\title{
Stability and stabilizability of 2D behaviors
}

\author{
Ettore Fornasini and Maria Elena Valcher \\ Dip. di Elettronica ed Informatica, Univ. di Padova, \\ via Gradenigo 6/a, 35131 Padova, Italy \\ e-mails: fornasini@dei.unipd.it,meme@dei.unipd.it
}

\begin{abstract}
In the paper stability and stabilizability properties are analysed in the context of two-dimensional (2D) behaviors. Searching for a comparison with traditional 2D input/output and state-space models, the interest is focused on linear, shift-invariant, complete behaviors whose autonomous part updates according to a quarter-plane causality law. For this class of behaviors, stabily and stabilizability definitions are introduced and related to the varieties of certain ideals, which can be obtained by suitably factorizing the Laurent polynomial matrices involved in the behavior description.
\end{abstract}

\section{Introduction}

The interest in two-dimensional system modelling is largely motivated by a number of physical processes, depending on a pair of independent variables, which make their appearance in several contexts such as seismology, X-ray image enhancement, image deblurring, digital picture processing, etc. The reasearch in this field was first concerned with two-dimensional input/output (i/o) and state-space descriptions [1, 3, 12], and in these settings the relevant stability issues, i.e. BIBO (bounded input bounded output) $[9,10]$ and internal [4] stability, have been fully investigated.

Stability definitions one refers to formalize the property of a system to produce trajectories which estinguish in the "future", provided that they satisfy certain regularity constraints in the "past". The underlying causality notion is quite obvious for ordinary 1D systems, and is based on the common interpretation of the independent variable $t$ as time coordinate. For $2 \mathrm{D}$ systems, instead, there is no natural ordering in $\mathbb{Z} \times \mathbb{Z}$, and hence no obvious future direction can be singled out in the system evolution. A reasonable choice, however, is the partial ordering of the discrete grid which corresponds to the quarter plane causality assumption. This induces a stability notion for $2 \mathrm{D}$ i/o maps and state models that refers to the trajectories evolution on the separation sets $\mathcal{S}_{t}:=\{(h, k) \in \mathbb{Z} \times \mathbb{Z}: h+k=t\}$ as $t$ goes to $+\infty$, and represents the setting under which
2D BIBO and internal stability have been investigated in the literature.

The behavioral approach was introduced in the eighties by J.C.Willems [13] for describing one-dimensional dynamical systems, and later extended to the multidimensional case $[6,11]$. Its distinguishing feature is that it focuses on the set of system trajectories, the behavior, while making no a priori distinction between inputs and outputs. Input/output and state space descriptions have to be deduced only later, from the mathematical equations, provided that the analysis of the behavior has enlightened some cause/effect structure.

In the $2 \mathrm{D}$ case a (discrete) dynamical system $\Sigma$ is a triple $\Sigma=\left(\mathbb{Z} \times \mathbb{Z}, \mathbb{R}^{q}, \mathfrak{B}\right)$, where $\mathbb{Z} \times \mathbb{Z}$ is the independent variables set, $\mathbb{R}^{q}$ the set of the values of system trajectories (the signal alphabet) and $\mathfrak{B} \subseteq\left(\mathbb{R}^{q}\right)^{\mathbb{Z} \times \mathbb{Z}}$ the set of admissible trajectories (the behavior). In order to make it possible a comparison with the previous models, we restrict our attention to linear, shiftinvariant and complete behaviors, namely behaviors that can be described by means of a set of $2 \mathrm{D}$ linear difference equations, or equivalently, as kernels of Laurent polynomial matrices. Furthermore, the stability/stabilizability definitions refer to a quarter plane causality structure.

The purpose of this contribution is to introduce these notions and to relate them to the algebraic features of the (Laurent) polynomial matrices involved in the behaviors descriptions. More precisely, section 2 deals with stability property of autonomous behaviors, while section 3 analyses the stabilizability problem for general (nonautonomous) behaviors.

Throughout the paper $\mathbb{R}\left[z_{1}, z_{2}\right], \mathbb{R}\left[z_{1}, z_{2}, z_{1}^{-1}, z_{2}^{-1}\right]$ and $\mathbb{R}\left(z_{1}, z_{2}\right)$ denote the rings of polynomials and Laurent polynomials (L-polynomials) and the field of rational functions, respectively, in the indeterminates $z_{1}$ and $z_{2}$, with coefficients in $\mathbb{R}$. Any two-dimensional sequences $\mathbf{w}=\{\mathbf{w}(h, k)\}_{h, k} \in \mathbb{Z} \in\left(\mathbb{R}^{q}\right)^{\mathbb{Z} \times \mathbb{Z}}$ will be identified with the corresponding formal power series $\sum_{h, k \in \mathbb{Z}} \mathbf{w}(h, k) z_{1}^{h} z_{2}^{k}$, and its support (or, equivalently, the support of the associated power series) will be defined as $\operatorname{supp}(\mathbf{w}):=\{(h, k) \in \mathbb{Z} \times \mathbb{Z}: \mathbf{w}(h, k) \neq$ $0\}$. For sake of brevity, the sequence space $\left(\mathbb{R}^{q}\right)^{\mathbb{Z} \times \mathbb{Z}}$ 
will be denoted by $\mathcal{R}_{\infty}^{q}$.

Given any nonzero L-polynomial $p$, let $z_{1}^{i} z_{2}^{j}$ be the (unique) monic monomial in $\mathbb{R}\left[z_{1}, z_{2}, z_{1}^{-1}, z_{2}^{-1}\right]$ such that $p^{*}:=z_{1}^{i} z_{2}^{j} p \in \mathbb{R}\left[z_{1}, z_{2}\right]$ and $\operatorname{supp}\left(p^{*}\right)$ intersects both coordinate axes in $\mathbb{Z} \times \mathbb{Z}$. The Laurent variety of $p, \mathcal{V}_{L}(p)$ is, by definition, the variety $\mathcal{V}\left(p^{*}\right)$ of $p^{*}$, i.e.

$$
\mathcal{V}_{L}(p):=\left\{(\alpha, \beta) \in \mathbb{C} \times \mathbb{C}: p^{*}(\alpha, \beta)=0\right\}
$$

Similarly, if $\mathcal{I}$ is a set of polynomials in $\mathbb{R}\left[z_{1}, z_{2}, z_{1}^{-1}, z_{2}^{-1}\right]$, in particular an ideal, its Laurent variety $\mathcal{V}_{L}(\mathcal{I})$ is the intersection of the Laurent varieties of all its elements.

Unless differently specified, all primeness properties of a matrix, even a strictly polynomial one, will refer to the ring of Laurent polynomials.

\section{Stability of autonomous behaviors}

Consider a dynamical system $\Sigma=\left(\mathbb{Z} \times \mathbb{Z}, \mathbb{R}^{q}, \mathfrak{B}\right)$, whose behavior $\mathfrak{B}$ is described as follows

$$
\mathfrak{B}=\operatorname{ker} H^{T}:=\left\{\mathbf{w} \in \mathcal{R}_{\infty}^{q}: H^{T} \mathbf{w}=0\right\},
$$

for some $m \times q$ matrix $H^{T}$, which can be assumed, without loss of generality, strictly polynomial.

The most significant property the behavior $\mathfrak{B}$ can be endowed with is undoubtely zero-controllability $[6,11]$ which expresses the possibility of "embedding" any portion of a behavior trajectory into a new one, whose support slightly exceeds that of the available portion. More precisely, we say that $\mathfrak{B}$ is zero-controllable if there exists a positive integer $\delta$ such that, for every finite set $\mathcal{T} \subset \mathbb{Z} \times \mathbb{Z}$ and every $\mathbf{w} \in \mathfrak{B}$, there is a trajectory $\tilde{\mathbf{w}} \in \mathfrak{B}$, which coincides with $\mathbf{w}$ in $\mathcal{T}$ and whose support is included in $\mathcal{T}^{\delta}:=\{(i, j) \in \mathbb{Z} \times \mathbb{Z}$ : $d((i, j), \mathcal{T})<\delta\}$, where the distance $d((i, j), \mathcal{T})$ is defined as min $\{|i-h|+|j-k| ;(h, k) \in \mathcal{T}\}$. Controllability induces a very peculiar polynomial matrix description for the behavior $\mathfrak{B}$. Indeed, $2 \mathrm{D}$ controllable behaviors are kernels of left factor prime L-polynomial matrices or, equivalently, image spaces of suitable L-polynomial operators [11], i.e. we have

$$
\mathfrak{B}=\operatorname{Im} G:=\left\{\mathbf{w} \in \mathcal{R}_{\infty}^{q}: \mathbf{w}=G \mathbf{u}, \mathbf{u} \in \mathcal{R}_{\infty}^{m}\right\},
$$

for some $G \in \mathbb{R}\left[z_{1}, z_{2}, z_{1}^{-1}, z_{2}^{-1}\right]^{q \times r}$.

A property which is somehow opposite to controllability is autonomy. A behavior $\mathfrak{B}$ is autonomous $[5,11]$ if there exists a solid cone $\mathcal{K}$ in $\mathbb{R} \times \mathbb{R}$, with vertex in the origin, such that the restriction $\left.\mathbf{w}\right|_{\mathcal{K}}$ of any behavior trajectory w to $\mathcal{K} \cap(\mathbb{Z} \times \mathbb{Z})$ allows to uniquely retrieve the remaining portion of $\mathbf{w}$. In the following, we will refer to $\mathcal{K}$ as to a characterizing cone of the behavior, and, with a slightly abuse of notation, we will use $\mathcal{K}$ to denote $\mathcal{K} \cap(\mathbb{Z} \times \mathbb{Z})$. Autonomous behaviors are kernels of full column rank L-polynomial matrices [11].

Controllable and autonomous behaviors constitute the building blocks for constructing all other behaviors, since every complete behavior $\mathfrak{B}$ can be expressed [5] as the sum $\mathfrak{B}=\mathfrak{B}_{c}+\mathfrak{B}_{a}$ of its "controllable part" $\mathfrak{B}_{c}$, i.e. the greatest controllable behavior included in $\mathfrak{B}$, and of a suitable autonomous behavior $\mathfrak{B}_{a}$.

Looking for possible comparisons with the stability property of quarter-plane causal state models considered in the literature, the stability definition we introduce is somehow tailored for analysing the trajectories on the halfplane $\mathcal{H}_{0}^{+}:=\{(h, k) \in \mathbb{Z} \times \mathbb{Z}: h+k>0\}$ and, in particular, on the positive orthant $\mathbb{N} \times \mathbb{N}$. Notice, however, that many alternative definitions could be given, and a more general approach to $2 \mathrm{D}$ behaviors stability is carried on in [7].

Intuitively speaking, a behavior $\mathfrak{B}$ is stable if every trajectory $\mathbf{w} \in \mathfrak{B}$ which is bounded in some sufficiently large region of the halfplane $\mathcal{H}_{0}^{-}:=\{(h, k) \in$ $\mathbb{Z} \times \mathbb{Z}: h+k \leq 0\}$ (the past) converges, at least, in the positive orthant (the future), i.e. $\|\mathbf{w}(h, k)\| \rightarrow 0$ as $h+k \rightarrow+\infty$. This immediately rules out all behaviors with a nontrivial controllable part $\mathfrak{B}_{c}$. In fact, as $\mathfrak{B}_{c}$ includes at least one finite support trajectory $\mathbf{w}_{f} \neq 0$, there exists a pair of positive integers $(\ell, m)$ such that $\sum_{i \in \mathbb{N}} z_{1}^{i \ell} z_{2}^{i m} \mathbf{w}_{f}$ is an infinite support trajectory which does not asymptotically estinguish. Consequently, stability notion concerns only autonomous behaviors and among them, by the previous assumptions on the system evolution, only those for which the cone $\mathcal{K}$, where the trajectories can be uniquely recognized [5], is included in $\mathcal{H}_{0}^{-}$. This is possible, in particular, when the behavior $\mathfrak{B}$ is described as the kernel of a polynomial matrix $H^{T} \in \mathbb{R}\left[z_{1}, z_{2}\right]^{m \times q}$ with $H^{T}(0,0)$ full column rank. For sake of simplicity, in this contribution we will afford only this special case and consider as characterizing cone any cone $\mathcal{K}$ which properly includes the negative orthant $\{(h, k) \in \mathbb{Z} \times \mathbb{Z}: h<$ $0, k<0\}$, is included in the halfplane $\mathcal{H}_{0}^{-}$and contains the halflines $\{(\ell,-\ell): \ell>0\}$ and $\{(\ell,-\ell): \ell<0\}$ as internal radii. It is immediately seen that $\mathbf{w} \mid \mathcal{K}$ uniquely determines the remaining portion of $\mathbf{w}$.

Definition 1 Let $\Sigma=\left(\mathbb{Z} \times \mathbb{Z}, \mathbb{R}^{q}, \mathfrak{B}\right)$ be a system, endowed with a complete autonomous behavior $\mathfrak{B}=$ $\operatorname{ker} H^{T}, H^{T} \in \mathbb{R}\left[z_{1}, z_{2}\right]^{m \times q}$ of rank $q$ and let $\mathcal{K}$ be a characterizing cone of $\mathfrak{B}$, that includes the negative orthant. We say that $\mathfrak{B}$ is $\mathcal{K}$-stable if every sequence $\mathbf{w} \in \mathfrak{B}$ bounded on the $\operatorname{strip}\left(\mathcal{K}+\operatorname{supp} H^{T}\right) \backslash(\mathcal{K}-$ $\left.\operatorname{supp} H^{T}\right)$ satisfies $\|\mathbf{w}(h, k)\| \rightarrow 0$ as $h+k \rightarrow+\infty$. Otherwise, $\mathfrak{B}$ is said to be $\mathcal{K}$-unstable. $\mathfrak{B}$ is stable if there exists a characterizing cone $\mathcal{K}$ such that $\mathfrak{B}$ is $\mathcal{K}$ stable. 
As in the case of state space models, stability of the autonomous behavior $\mathfrak{B}=\operatorname{ker} H^{T}$ can be related to the intersections of the algebraic variety of the maximal order minors of $H^{T}$ with the closed unit polydisk

$$
\overline{\mathcal{P}}_{1}:=\left\{\left(z_{1}, z_{2}\right) \in \mathbb{C} \times \mathbb{C}:\left|z_{1}\right| \leq 1,\left|z_{2}\right| \leq 1\right\} .
$$

Proposition 1 Let $\Sigma=\left(\mathbb{Z} \times \mathbb{Z}, \mathbb{R}^{q}, \mathfrak{B}\right)$ be a system, endowed with a complete autonomous behavior $\mathfrak{B}=\operatorname{ker} H^{T}$, and assume that $H^{T}$ is an $m \times q$ (strictly) polynomial matrix, with $H^{T}(0,0)$ full column rank. $\mathfrak{B}$ is stable if and only if the variety $\mathcal{V}_{L}\left(H^{T}\right)$ of the maximal order minors of $H^{T}$ does not intersect the closed unit polydisk $\overline{\mathcal{P}}_{1}$.

Proof Let $\mathcal{K}$ be a characterizing cone of $\mathfrak{B}$, which fulfills the aforementioned constraints. If $\mathcal{V}_{L}\left(H^{T}\right) \cap$ $\overline{\mathcal{P}}_{1}=\emptyset$, there exists also $\rho>1$ such that $\mathcal{V}_{L}\left(H^{T}\right) \cap \overline{\mathcal{P}}_{\rho}=$ $\emptyset$, with $\overline{\mathcal{P}}_{\rho}:=\left\{\left(z_{1}, z_{2}\right) \in \mathbb{C} \times \mathbb{C}:\left|z_{1}\right| \leq \rho,\left|z_{2}\right| \leq \rho\right\}$. Thus, the ideal generated in $\mathbb{R}\left[z_{1}, z_{2}, z_{1}^{-1}, z_{2}^{-1}\right]$ by the maximal order minors $m_{i}$ of $H^{T}$ includes a polynomial $p_{\rho}=\sum_{i} c_{i} m_{i} \in \mathbb{R}\left[z, z_{2}\right]$ whose variety $\mathcal{V}\left(p_{\rho}\right)$ does not intersect $\overline{\mathcal{P}}_{\rho}$ (and hence, in particular, $p_{\rho}(0,0) \neq 0$ ).

If $S_{i}$ denotes the selection matrix corresponding to $m_{i}$, then $m_{i} I=\operatorname{adj}\left(S_{i} H^{T}\right) S_{i} H^{T}$, and consequently

$$
L:=\sum_{i} c_{i} \operatorname{adj}\left(S_{i} H^{T}\right) S_{i}
$$

is an L-polynomial matrix satisfying $L H^{T}=p_{\rho} I$. Consider a trajectory $\mathbf{w} \in \mathfrak{B}$ which is bounded on $\left(\mathcal{K}+\operatorname{supp} H^{T}\right) \backslash\left(\mathcal{K}-\operatorname{supp} H^{T}\right)$, and let $\mathbf{w}_{-}$and $\mathbf{w}_{+}$ denote the restrictions of $\mathbf{w}$ to $\mathcal{K}$ and $(\mathbb{Z} \times \mathbb{Z}) \backslash \mathcal{K}$, respectively. Since $H^{T}\left(\mathbf{w}_{+}+\mathbf{w}_{-}\right)=0$, the sequence $\mathbf{s}:=H^{T} \mathbf{w}_{+}=-H^{T} \mathbf{w}_{-}$, and hence also $\tilde{\mathbf{s}}:=L \mathbf{s}$, have supports included in some suitable strip around the generating lines of $\mathcal{K}$. So, two integer pairs $\left(h_{1}, k_{1}\right)$ and $\left(h_{2}, k_{2}\right)$ can be found, with $h_{1}<0<h_{2}$ and $k_{1}<0<k_{2}$, such that the support of $\tilde{s}$ is included in

$$
\mathcal{T}:=\left(\left(h_{2}, k_{2}\right)+\mathcal{K}\right) \backslash\left(\left(h_{1}, k_{1}\right)+\mathcal{K}\right),
$$

and is bounded therein.

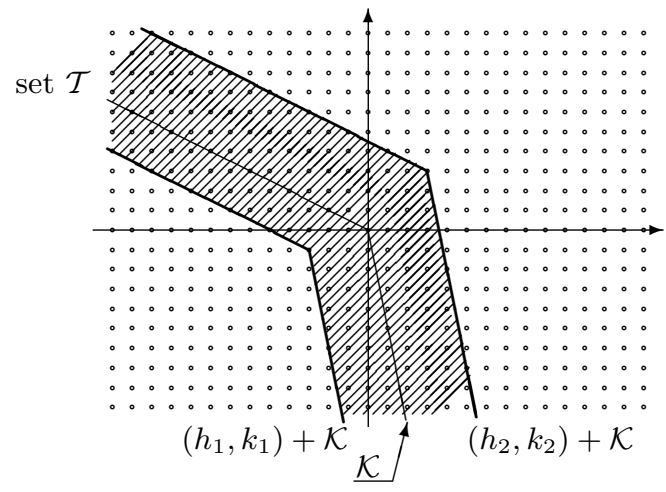

Set, now, $S:=\sup \{\|\tilde{\mathbf{s}}(i, j)\|,(i, j) \in \mathcal{T}\}$. From $\tilde{\mathbf{s}}=$ $L H^{T} \mathbf{w}_{+}=p_{\rho} \mathbf{w}_{+}$, it follows that the sequence $\mathbf{w}_{+}=$ $\tilde{\mathbf{s}} / p_{\rho}$ satisfies

$$
\mathbf{w}_{+}(h, k)=\sum_{(i, j) \text { s.t. }(h-i, k-j) \in \mathcal{T}} \tilde{s}(h-i, k-j) q_{i j} .
$$

where $q_{i j}$ are the coefficients of the power series expansion $1 / p_{\rho}=\sum_{i, j \in \mathbb{N}} q_{i, j} z_{1}^{i} z_{2}^{j}$ which is absolutely convergent in $\overline{\mathcal{P}}_{\rho}$. As a consequence, there exists a positive real $K$ such that

$$
\sum_{i+j=t}\left|q_{i j}\right|<K / \rho^{t}, \quad \forall t \in \mathbb{N} .
$$

and for $\left.(h, k) \in \mathcal{H}_{0}^{+}\right\}$, with $h+k$ sufficiently large, we have

$$
\begin{aligned}
\|\mathbf{w}(h, k)\| & =\left\|\mathbf{w}_{+}(h, k)\right\| \\
& \leq \sum_{(i, j) \text { s.t. }(h-i, k-j) \in \mathcal{T}}^{h+k(h-i, k-j)|| q_{i j} \mid} \\
& \leq S \sum_{i+j=h+k-h_{2}-k_{2}}^{h+k k_{i j} \mid} \\
& \leq S K \sum_{t=h+k-h_{2}-k_{2}}^{h+k-h_{1}-k_{1}} \frac{1}{\rho^{t}} .
\end{aligned}
$$

Therefore, $\|\mathbf{w}(h, k)\| \rightarrow 0$ as $h+k \rightarrow+\infty$, thus proving stability.

Suppose, now, that $\mathcal{V}\left(\bar{H}^{T}\right)$ intersects $\overline{\mathcal{P}}_{1}$ in some point $(\alpha, \beta)$, with $|\alpha| \leq 1$ and $|\beta| \leq 1$. Notice that, by the assumption on $H^{\bar{T}}(0,0),(\alpha, \bar{\beta}) \neq(0,0)$. Then $\bar{H}^{T}(\alpha, \beta)$ has not full column rank and there exists some nonzero vector $\mathbf{u}$ belonging to its kernel. Consider the (complex valued) trajectory $\mathbf{w}$ defined by:

$$
\mathbf{w}(h, k)=\frac{1}{\alpha^{h}} \frac{1}{\beta^{k}} \mathbf{u}, \quad \forall(h, k) \in \mathbb{Z} \times \mathbb{Z},
$$

if $\alpha$ and $\beta$ are both nonzero,

$$
\mathbf{w}(h, k)=\left\{\begin{array}{ll}
\beta^{-k} \mathbf{u}, & \text { if } h=0 \\
0 & \text { elsewhere }
\end{array} \quad \forall(h, k) \in \mathbb{Z} \times \mathbb{Z},\right.
$$

if $\alpha=0$, and similarly if $\beta=0$. The trajectory $\mathbf{w}$ satisfies $H^{T} \mathbf{w}=0$, is bounded on a suitable characterizing cone $\mathcal{K}$ which properly includes the negative orthant and does not includes the halflines $\{(\ell,-\ell): \ell>0\}$ and $\{(\ell,-\ell): \ell<0\}$. Finally, $\|\mathbf{w}(h, k)\|$ is not infinitesimal as $h+k$ goes to infinity. Thus either the real part or the immaginary part of $\mathbf{w}$ represents a vector sequence in $\mathfrak{B}$, bounded in $\mathcal{K}$ and not asymptotically estinguishing in the positive orthant, thus proving that $\mathfrak{B}$ is not stable.

\section{Stabilizability of nonautonomous behaviors}

As previously remarked, any complete behavior $\mathfrak{B}=$ $\operatorname{ker} H^{T}$, can be represented as the sum of its controllable part $\mathfrak{B}_{c}$ and of an autonomous behavior $\mathfrak{B}_{a}$. The 
controllable part $\mathfrak{B}_{c}$ is easily computed upon factorizing $H^{T}$ as $H^{T}=F \bar{H}^{T}, F$ a full column rank and $\bar{H}^{T}$ a left factor prime L-polynomial matrix, respectively. Indeed, $\mathfrak{B}_{c}$ coincides with the kernel of $\bar{H}^{T}$. On the other hand, $\mathfrak{B}_{a}$ can be selected with a certain degree of freedom but, differently from the $1 \mathrm{D}$ case, in general it is not possible to guarantee that $\mathfrak{B}_{c}+\mathfrak{B}_{a}$ is a direct sum $[5]$.

As a result of the above representation, every trajectory $\mathbf{w}$ of $\mathfrak{B}$ can be thought of as the sum of a trajectory $\mathbf{w}_{c} \in \mathfrak{B}_{c}$ and of a trajectory $\mathbf{w}_{a} \in \mathfrak{B}_{a}$. Replace now $\mathbf{w}_{c}$ with a trajectory $\mathbf{w}_{c}^{\prime} \in \mathfrak{B}_{c}$ which coincides with $\mathbf{w}_{c}$ in $\mathcal{H}_{0}^{-}$and is zero for all $(h, k)$ with $h+k \geq \delta, \delta$ a suitable nonnegative integer. If we assume, as before, that every trajectory of the autonomous part is uniquely determined by its restriction to the halfplane $\mathcal{H}_{0}^{-}$, it is immediate to realize that the possibility of asymptotically driving to zero a behavior trajectory only depends on the autonomous part $\mathfrak{B}_{a}$. These arguments lead to the following definition of stabilizability.

Definition 2 Let $\Sigma=\left(\mathbb{Z} \times \mathbb{Z}, \mathbb{R}^{q}, \mathfrak{B}\right)$ be a system, endowed with a complete behavior $\mathfrak{B}=\operatorname{ker} H^{T}, H^{T}$ an $m \times q$ polynomial matrix, and let $\mathfrak{B}_{c}$ be the controllable part of $\mathfrak{B}$. We say that $\mathfrak{B}$ is stabilizable if there exists a decomposition $\mathfrak{B}=\mathfrak{B}_{c}+\mathfrak{B}_{a}, \mathfrak{B}_{a}=\operatorname{ker} H_{a}^{T}$ autonomous with characteristic cone $\mathcal{K}$, such that for every sequence $\mathbf{w} \in \mathfrak{B}$, which can be expressed as $\mathbf{w}=\mathbf{w}_{c}+\mathbf{w}_{a}$, with $\mathbf{w}_{c} \in \mathfrak{B}_{c}$ and $\mathbf{w}_{a} \in \mathfrak{B}_{a}$ bounded on the strip $\left(\mathcal{K}+\operatorname{supp} H_{a}^{T}\right) \backslash\left(\mathcal{K}-\operatorname{supp} H_{a}^{T}\right)$, there exists $\tilde{\mathbf{w}} \in \mathfrak{B}$, which coincides with $\mathbf{w}$ on the halfplane $\mathcal{H}_{0}^{-}$ and satisfies $\|\tilde{\mathbf{w}}(h, k)\| \rightarrow 0$ as $h+k \rightarrow+\infty$.

It is easily seen that stabilizability is equivalent to the possibility of expressing $\mathfrak{B}$ as the sum of its controllable part and of a stable autonomous behavior. Consequently, the following proposition provides a characterization of stabilizability.

Proposition 2 Let $\Sigma=\left(\mathbb{Z} \times \mathbb{Z}, \mathbb{R}^{q}, \mathfrak{B}\right)$ be a system, endowed with a complete behavior $\mathfrak{B}=\operatorname{ker} H^{T}$, and assume that $H^{T} \in \mathbb{R}\left[z_{1}, z_{2}\right]^{m \times q}$ can be expressed as $H^{T}=F \bar{H}^{T}$, for some polynomial matrix $F$, with $F(0,0)$ full column rank, and some left factor prime polynomial matrix $\bar{H}^{T}$. $\mathfrak{B}$ can be expressed as

$$
\mathfrak{B}=\mathfrak{B}_{a}+\mathfrak{B}_{c}
$$

for some stable autonomous behavior $\mathfrak{B}_{a}$, if and only if $\mathcal{V}_{L}(F) \cap \overline{\mathcal{P}}_{1}=\emptyset$.

Proof By Proposition 1, we need to prove that $\mathcal{V}_{L}(F) \cap \overline{\mathcal{P}}_{1}=\emptyset$ is a necessary and sufficent condition for the existence of a polynomial matrix $H_{a}^{T}$, with $H_{a}^{T}(0,0)$ full column rank and $\mathcal{V}_{L}\left(H_{a}^{T}\right) \cap \overline{\mathcal{P}}_{1}=\emptyset$, such that $\mathfrak{B}=\operatorname{ker} H_{a}^{T}+\mathfrak{B}_{c}$.

Assume, first, that $\mathcal{V}_{L}(F) \cap \overline{\mathcal{P}}_{1}=\emptyset$. Then, there exists a polynomial matrix $X$ such that

$$
s I_{r}=X F,
$$

for some polynomial $s \in \mathbb{R}\left[z_{1}, z_{2}\right]$, belonging to the ideal (in $\mathbb{R}\left[z_{1}, z_{2}, z_{1}^{-1}, z_{2}^{-1}\right]$ ) of the maximal order minors of $F$ and satisfying $\mathcal{V}_{L}(s) \cap \overline{\mathcal{P}}_{1}=\emptyset$ and, in particular, $s(0,0) \neq 0$. Introduce the behavior $\overline{\mathfrak{B}}:=$ $\operatorname{ker}\left(s \bar{H}^{T}\right)$. Since

$$
\operatorname{ker}\left(s \bar{H}^{T}\right)=\operatorname{ker}\left(X F \bar{H}^{T}\right)=\operatorname{ker}\left(X H^{T}\right) \supseteq \operatorname{ker} H^{T},
$$

it follows that $\overline{\mathfrak{B}} \supseteq \mathfrak{B}$. Furthermore, it is easily seen that the behavior $\overline{\mathfrak{B}}_{c}+\operatorname{ker}\left(s I_{q}\right)$ coincides with the set of all trajectories $\mathbf{w}$ such that

$$
\left[\begin{array}{c}
0 \\
0 \\
I_{q}
\end{array}\right] \mathbf{w}=\left[\begin{array}{ccc}
\bar{H}^{T} & \mid & 0 \\
\hline 0 & \mid & s I_{q} \\
\hline I_{q} & \mid & I_{q}
\end{array}\right]\left[\begin{array}{c}
\overline{\mathbf{w}} \\
\mathbf{w}_{a}
\end{array}\right], \quad \exists \overline{\mathbf{w}}, \mathbf{w}_{a},
$$

and since

$$
T:=\left[\begin{array}{llll}
-s I_{r} & \mid & -\bar{H}^{T} & \mid s \bar{H}^{T}
\end{array}\right]
$$

is a left factor prime matrix, of size $r \times(r+2 q)$, whose rows are orthogonal to the columns of

$$
R:=\left[\begin{array}{ccc}
\bar{H}^{T} & \mid & 0 \\
\hline 0 & & s I_{q} \\
\hline I_{q} & \mid & I_{q}
\end{array}\right] \in \mathbb{R}\left[z_{1}, z_{2}\right]^{(r+2 q) \times 2 q},
$$

it follows that $\operatorname{Ker} T=\operatorname{Im} R$ and hence

$$
\mathfrak{B}_{c}+\operatorname{ker}\left(s I_{q}\right)=\left\{\mathbf{w}:\left[\begin{array}{c}
0 \\
0 \\
I_{q}
\end{array}\right] \mathbf{w} \in \operatorname{ker} T\right\}=\overline{\mathfrak{B}} .
$$

Consequently,

$$
\begin{aligned}
\mathfrak{B} & =\mathfrak{B} \cap \overline{\mathfrak{B}}=\left(\mathfrak{B} \cap \mathfrak{B}_{c}\right)+\left(\mathfrak{B} \cap \operatorname{ker}\left(s I_{q}\right)\right) \\
& =\mathfrak{B}_{c}+\left(\mathfrak{B} \cap \operatorname{ker}\left(s I_{q}\right)\right)=\mathfrak{B}_{c}+\operatorname{ker}\left[\begin{array}{c}
s I_{q} \\
H^{T}
\end{array}\right],
\end{aligned}
$$

and the result holds true for $H_{a}^{T}:=\left[\begin{array}{c}s I_{q} \\ H^{T}\end{array}\right]$.

Suppose, now, that (5) holds true for some stable autonomous behavior $\mathfrak{B}_{a}=\operatorname{ker} H_{a}^{T}$, with $H_{a}^{T} \in$ $\mathbb{R}\left[z_{1}, z_{2}\right]^{l \times q}$ full column rank and satisfying $\mathcal{V}_{L}\left(H_{a}^{T}\right) \cap$ $\overline{\mathcal{P}}_{1}=\emptyset$. This implies that $\mathfrak{B}$ can be expressed as

$\mathfrak{B}=\left\{\mathbf{w}:\left[\begin{array}{c}0 \\ 0 \\ I_{q}\end{array}\right] \mathbf{w}=\left[\begin{array}{cc}\bar{H}^{T} & 0 \\ 0 & H_{a}^{T} \\ I_{q} & I_{q}\end{array}\right]\left[\begin{array}{c}\overline{\mathbf{w}} \\ \mathbf{w}_{a}\end{array}\right], \exists \overline{\mathbf{w}}, \mathbf{w}_{a}\right\}$.

Let $\left[\begin{array}{lll}X & Y & Z\end{array}\right]$ be an $(l+r-q) \times(l+r+q)$ left factor prime matrix whose rows are orthogonal to the columns of

$$
R:=\left[\begin{array}{cc}
\bar{H}^{T} & 0 \\
0 & H_{a}^{T} \\
I_{q} & I_{q}
\end{array}\right]
$$


Clearly, $\mathfrak{B} \equiv \operatorname{ker} Z \equiv \operatorname{ker}\left(X \bar{H}^{T}\right)$. We aim to show that $X$ is a full column rank matrix, with $\mathcal{V}_{L}(X) \cap \overline{\mathcal{P}}_{1}=\emptyset$. The full column rank condition follows immediately from the property that all generator matrices of $\mathfrak{B}$ share the same rank. Moreover, as a consequence of Corollary A.2, every $(l+r-q)$ th order minor of $\left[\begin{array}{lll}X & Y & Z\end{array}\right]$ which includes all the columns of $X$ must divide some suitable $r$ th order minor of $H_{a}^{T}$. This guarantees that $\mathcal{V}_{L}(X)$ cannot intersect the closed unit polydisk. Since $\mathfrak{B}=\operatorname{ker}\left(X \bar{H}^{T}\right)=\operatorname{ker}\left(F \bar{H}^{T}\right)$, the result is, now, an immediate consequence of Lemma A.3.

REMARK In the context of $1 \mathrm{D}$ behaviors, important properties like controllability and stabilizability are captured by the characteristic polynomial $\chi$ of the behavior [14]. Given any kernel description of the behavior, $\mathfrak{B}=\operatorname{ker} H^{T}$, with $H^{T}$ of rank $r, \chi$ can be uniquely recovered (modulo some unit in $\mathbb{R}\left[z, z^{-1}\right]$ ) as the g.c.d. of the $r$ th order minors of $H^{T}$, and it is has been proved that $\mathfrak{B}$ is controllable if and only if $\chi$ is unit in $\mathbb{R}\left[z, z^{-1}\right]$, while it is stabilizable if and only if $\chi$ is Hurwitz.

If $\mathfrak{B}=\operatorname{ker} H^{T}$ is any $2 \mathrm{D}$ behavior and $H^{T}$ is expressed as $H^{T}=F \bar{H}^{T}$, for some L-polynomial matrix $F$ and some left factor prime matrix $\bar{H}^{T}$, it has been shown that $\mathfrak{B}$ is controllable if and only if $F$ is right zero prime, and hence $\mathcal{V}_{L}(F)=\emptyset$, while is stabilizable iff $\mathcal{V}_{L}(F) \cap \overline{\mathcal{P}}_{1}=\emptyset$. This suggests that, in the $2 \mathrm{D}$ case, the notion of characteristic polynomial of a behavior $\mathfrak{B}$ should be replaced by that of characteristic variety, $\mathcal{V}_{L}(\mathfrak{B})$, which can be obtained from the aforementioned decomposition of any matrix $H^{T}$ such that $\mathfrak{B}=\operatorname{ker} H^{T}$ and it coincides with $\mathcal{V}_{L}(F)$. Notice that, as proved in Lemma A.3, such a variety is uniquely determined and does not depend on the particular matrix adopted for the behavior description.

To conclude, it is worthwhile underlying that, differently from the $1 \mathrm{D}$ case, the information about the variety $\mathcal{V}_{L}(\mathfrak{B})$ cannot be captured by a single "characteristic polynomial". This is immediately apparent when considering varieties which consist of a finite number of points. Finally, $\mathcal{V}_{L}(\mathfrak{B})$ does not coincide with the variety of the maximal order minors of $H^{T}$, and this is due to the fact that the variety of $\bar{H}^{T}$, which is related to the controllable part of $\mathfrak{B}$, does not affect stabilizability.

\section{Appendix}

Lemma A.1 Let $T \in \mathbb{R}\left[z_{1}, z_{2}, z_{1}^{-1}, z_{2}^{-1}\right]^{k \times n}$ and $R \in \mathbb{R}\left[z_{1}, z_{2}, z_{1}^{-1}, z_{2}^{-1}\right]^{n \times(n-k)}$ be two L-polynomial matrices, left and right factor prime, respectively, satisfying $T R=0$.
If $m_{i}(T)$ denotes the ith maximal $(=k$ th) order minor of $T$ and $m_{i}(R)$ the maximal $(=(n-k)$ th) order minor of $R$, obtained by selecting in $R$ rows of complementary indices w.r.t. the columns selected in $T$, then $m_{i}(T)$ and $m_{i}(R)$ coincide modulo some unit in $\mathbb{R}\left[z_{1}, z_{2}, z_{1}^{-1}, z_{2}^{-1}\right]$.

Proof Since $T$ is left factor prime, there exist two Lpolynomial matrices, $X_{1}$ and $X_{2}$, and two polynomials, $g_{1}\left(z_{1}\right) \in \mathbb{R}\left[z_{1}\right]$ and $g_{2}\left(z_{2}\right) \in \mathbb{R}\left[z_{2}\right]$, such that

$$
T X_{1}=g_{1}\left(z_{1}\right) I_{k} \quad \text { and } \quad T X_{2}=g_{2}\left(z_{2}\right) I_{k} .
$$

Consider for instance $m_{1}(T)$, the maximal order minor of $T$ corresponding to the selection of the first $k$ columns of $T$. Complete $T$ into a square matrix by resorting to a $(n-k) \times n$ matrix, whose columns are all zero except for the last $n-k$, which consitute the identity matrix. Thus

$$
\left[\begin{array}{ccc} 
& T & \\
0 & & I_{n-k}
\end{array}\right]\left[X_{1} \mid R\right]=\left[\begin{array}{cc}
g_{1}\left(z_{1}\right) I_{k} & 0 \\
Q & M_{1}(R)
\end{array}\right],
$$

where $M_{1}(R)$ is the $(n-k) \times(n-k)$ submatrix of $R$ obtained by selecting the last $n-k$ rows. Assuming $R_{1}:=\left[\begin{array}{ll}X_{1} & R\end{array}\right]$ and $\mu_{1}:=\operatorname{det} M_{1}(R)$, we get

$$
m_{1}(T) \operatorname{det} R_{1}=\left[g_{1}\left(z_{1}\right)\right]^{k} \mu_{1}(R) .
$$

Now replace $X_{2}$ with $X_{1}$ in (6) and let $R_{2}:=\left[\begin{array}{ll}X_{2} & R\end{array}\right]$. We obtain

$$
m_{1}(T) \operatorname{det} R_{2}=\left[g_{2}\left(z_{2}\right)\right]^{k} \mu_{1}(R) .
$$

So $m_{1}(T) \mid\left[g_{1}\left(z_{1}\right)\right]^{k} \mu_{1}(R)$ and $m_{1}(T) \mid\left[g_{2}\left(z_{2}\right)\right]^{k} \mu_{1}(R)$. Since $\left[g_{1}\left(z_{1}\right)\right]^{k}$ and $\left[g_{2}\left(z_{2}\right)\right]^{k}$ are coprime, then

$$
m_{1}(T) \mid \mu_{1}(R) \text {. }
$$

Dually, as $R$ is right factor prime, there exist two Lpolynomial matrices, $Y_{1}$ and $Y_{2}$, and two polynomials, $h_{1}\left(z_{1}\right) \in \mathbb{R}\left[z_{1}\right]$ and $h_{2}\left(z_{2}\right) \in \mathbb{R}\left[z_{2}\right]$, such that

$$
Y_{1} R=h_{1}\left(z_{1}\right) I_{n-k} \quad \text { and } \quad Y_{2} R=h_{2}\left(z_{2}\right) I_{n-k} .
$$

We can proceed as before, getting

$$
\left[\begin{array}{c}
T \\
Y_{1}
\end{array}\right]\left[\begin{array}{ll}
I_{k} & \\
& R \\
0 &
\end{array}\right]=\left[\begin{array}{cc}
M_{1}(T) & 0 \\
T & h_{1}\left(z_{1}\right) I_{n-k}
\end{array}\right],
$$

where $M_{1}(T)$ is the $k \times k$ submatrix of $T$ obtained by selecting its first $k$ columns. Assuming $S_{1}:=\left[\begin{array}{c}T \\ Y_{1}\end{array}\right]$, we get $\operatorname{det} S_{1} \mu_{1}(R)=m_{1}(T)\left[h_{1}\left(z_{1}\right)\right]^{n-k}$, and, analogously, $\operatorname{det} S_{2} \mu_{1}(R)=m_{1}(T)\left[h_{2}\left(z_{2}\right)\right]^{n-k}$, where $S_{2}:=$ $\left[\begin{array}{c}T \\ Y_{2}\end{array}\right]$

Therefore $\mu_{1}(R)$ is a common factor of $m_{1}(T)\left[h_{1}\left(z_{1}\right)\right]^{n-k}$ and $m_{1}(T)\left[h_{2}\left(z_{2}\right)\right]^{n-k}$, and then

$$
\mu_{1}(R) \mid m_{1}(T)
$$


(7) and (8) together imply that $m_{1}(T)$ and $\mu_{1}(R)$ differ in a unit of $\mathbb{R}\left[z_{1}, z_{1}^{-1}, z_{2}, z_{2}^{-1}\right]$.

Similarly, we can show that the same result holds for any other pair of corresponding minors in $T$ and in $R$.

Corollary A.2 Let $T \in \mathbb{R}\left[z_{1}, z_{2}, z_{1}^{-1}, z_{2}^{-1}\right]^{k \times n}$ and $R \in \mathbb{R}\left[z_{1}, z_{2}, z_{1}^{-1}, z_{2}^{-1}\right]^{n \times(n-k)}$ be L-polynomial matrices, $T$ left factor prime and $R$ full column rank, which satisfy $T R=0$, and let $r \in \mathbb{R}\left[z_{1}, z_{2}\right]$ denote a g.c.d. of the maximal order minors of $R$.

If $m_{i}(T)$ denotes the ith maximal order minor of $T$ and $m_{i}(R)$ the maximal order minor of $R$, obtained by selecting the rows of $R$ of complementary indices, then $m_{i}(T)$ coincides with $m_{i}(R) / r$ modulo some unit in $\mathbb{R}\left[z_{1}, z_{2}, z_{1}^{-1}, z_{2}^{-1}\right]$.

Proof Follows immediately from Lemma A.1, upon factorizing $R$ as $R=\bar{R} \Delta$, with $\bar{R}$ right factor prime and $\Delta$ nonsingular square. Clearly, $\operatorname{det} \Delta=c \cdot r, c$ a unit in $\mathbb{R}\left[z_{1}, z_{2}, z_{1}^{-1}, z_{2}^{-1}\right]$.

Lemma A.3 Let $\bar{H}_{i}^{T}, i=1,2$, be left prime $L$ polynomial matrix, and let $X$ and $F$ be full column rank L-polynomial matrices. If $\operatorname{ker}\left(F \bar{H}_{1}^{T}\right)=$ $\operatorname{ker}\left(X \bar{H}_{2}^{T}\right)$, then $\bar{H}_{1}^{T}=U \bar{H}_{2}^{T}$ for some unimodular matrix $U$ and $\mathcal{V}_{L}(F)=\mathcal{V}_{L}(X)$.

Proof Condition $\operatorname{ker}\left(F \bar{H}_{1}^{T}\right)=\operatorname{ker}\left(X \bar{H}_{2}^{T}\right)$ implies

$$
P\left(F \bar{H}_{1}^{T}\right)=X \bar{H}_{2}^{T} \quad Q\left(X \bar{H}_{2}^{T}\right)=F \bar{H}_{1}^{T},
$$

for suitable L-polynomial matrices $P$ and $Q$. Since $F$ and $X$ have full column rank, they admit left inverses $F^{-1}$ and $X^{-1}$, and we get

$$
\left(X^{-1} P F\right) \bar{H}_{1}^{T}=\bar{H}_{2}^{T} \quad\left(F^{-1} Q X\right) \bar{H}_{2}^{T}=\bar{H}_{1}^{T} .
$$

This implies that $\bar{H}_{1}^{T}$ and $\bar{H}_{2}^{T}$ have the same rank and, being left factor prime, they must differ [6] in some L-polynomial unimodular factor $U:=F^{-1} Q X$. Consequently, (9) can be rewritten as

$$
P\left(F \bar{H}_{1}^{T}\right)=X U \bar{H}_{1}^{T} \quad Q\left(X U \bar{H}_{1}^{T}\right)=F \bar{H}_{1}^{T} .
$$

Since $\bar{H}_{1}^{T}$ is full row rank, then $P F=X U$ and $Q X U=$ $F$, thus implying both $\mathcal{V}_{L}(X)=\mathcal{V}_{L}(X U) \subseteq \mathcal{V}_{L}(F)$ and $\mathcal{V}_{L}(F) \subseteq \mathcal{V}_{L}(X U)=\mathcal{V}_{L}(X)$. This proves the result.

\section{References}

[1] S. Attasi. Systèmes linéaires homogènes à deux indices. Rapport LABORIA, 31, 1973.

[2] E. Fornasini. A note on output feedback stabilizability of multivariable 2-D systems. Systems $\&$ Control Letters, 10, pp.45-50, 1988.
[3] E. Fornasini and G. Marchesini. Doubly indexed dynamical systems. Math. Sys. Theory, 12, pp.59-72, 1978.

[4] E. Fornasini and G. Marchesini. Stability analysis of 2D systems. IEEE Trans. Circ. and Sys., CAS-32, pp.1246-54, 1980.

[5] E. Fornasini, P. Rocha, and S. Zampieri. State realization of 2D finite dimensional autonomous systems. SIAM J. Contr. Optimiz., 31, pp.1502-1517, 1993.

[6] E. Fornasini and M.E. Valcher. Algebraic aspects of $2 \mathrm{D}$ convolutional codes. IEEE Trans. Info. Th., IT 33, pp.1210-1225, 1994.

[7] E. Fornasini and M.E. Valcher. Stability and stabilizability properties in the context of $2 \mathrm{D}$ behaviors. in preparation, 1997.

[8] W. Fulton. Algebraic curves. W.A.Benjamin, 1969.

[9] D. Goodman. Some stability properties of twodimensional linear shift-invariant digital filters. IEEE Trans. Circ. Sys., CAS-24, pp.201-208, 1977.

[10] T.S. Huang. Stability of two-dimensional recursive filters. IEEE Trans. Audio Electroacoustics, AU20, pp.158-163, 1972.

[11] P. Rocha. Structure and Representation of 2-D Systems. PhD thesis, University of Groningen, The Netherlands, 1990.

[12] R.P. Roesser. A discrete state space model for linear image processing. IEEE Trans. Aut.Contr., AC 20, pp.1-10, 1975.

[13] J.C. Willems. From time series to linear system, part I, pp. Finite dimensional linear time invariant systems. Automatica, 22, pp.561-580, 1986.

[14] J.C. Willems. On interconnections, control, and feedback. IEEE Trans. Aut. Contr., AC 42, pp.326339, 1997. 